\title{
Human milk bank
}

Keywords: infant feeding, pasteurized, human milk, morbidities, infections, breast milk, sick neonates, biological mother, postpartum, electric pumps

\section{Human milk bank}

Breastfeeding is the best method of infant feeding because human milk continues to be the only milk which is tailor-made and uniquely suited to the human infant. When a mother, for some reason, is unable to feed her infant directly, her breast milk should be expressed and fed to the infant. If mother's own milk is unavailable or insufficient, the next best option is to use pasteurized donor human milk (PDHM). India faces its own unique challenges, having the highest number of low birth weight babies, and significant mortality and morbidity in very low birth weight (VLBW) population. In our country, the burden of low birth weight babies in various hospitals is about $20 \%$ with significant mortality and morbidities. Feeding these babies with breast milk can significantly reduce the risk of infections.

\section{History}

Donation of breast milk from one woman to an unrelated infant has a long history. Before this century, the infant would have been directly breastfed by the woman who was referred to as a "wet nurse". Rules governing wet nursing have been around since 1800 BC. Human milk banking had peaks and troughs. In the early half of $20^{\text {th }}$ century, milk banking saw resurgence in popularity, but around the 1970s, this began to change. The first reason for this loss of interest in human milk was the heavy promotion of infant formula, including formulas specially designed for preterm infants. Later, a fear of transmission of viruses, including HIV, in body fluids led to an anxiety about donation of body fluids, including breast milk.

\section{Uses}

Banked human milk is regarded as "the next best" after the biological mother's breast milk. It is used for the treatment of many conditions (mainly in Neonatal Intensive Care Units: NICUs): prematurity, malabsorption, short-gut syndrome, intractable diarrhea, nephrotic syndrome, some congenital anomalies, formula intolerance, failure to thrive, immune deficiencies (IgA). Studies have found that breast milk has a protective effect against necrotisingenterocolitis (NEC).

\section{Asia's first human milk bank}

The first milk bank in Asia under the name of Sneha, founded by Dr. Armeda Fernandez, was started in Dharavi, Mumbai on November 27, 1989. Currently, the number of human milk banks (HMB) has grown to nearly 14 all over India but the growth of human milk banks has been very slow as compared to the growth of neonatal intensive care units. One of the major reasons for loss of interest in human milk banking was the promotion of formula milk by the industry. Keeping in mind the complications associated with formula feeding to the sick, tiny preterm neonates and mothers' inability to breastfeed in the initial period, there is a need to establish human milk banks in all level II and level III facilities.
Volume 5 Issue 6 - 2018

\author{
Ansa Sunil \\ Department of Nursing, Jubilee Mission College of Nursing,
} India

Correspondence: Ansa Sunil, Department of Nursing, Jubilee Mission College of Nursing, Thrissur, Kerala, India, Tel 9447763348,Email ansesunill6@gmail.com

Received: May 03, 2017 | Published: November 23, 2018

\section{International statement}

WHO and UNICEF, made a joint statement in 1980: "Where it is not possible for the biological mother to breast feed, the first alternative, if available, should be the use of human milk from other sources. Human milk banks should be made available intop appropriate situations".

\section{Guidelines for human milk bank}

\section{Location}

Human milk banks are primarily focused to provide donor milk to high risk newborns admitted in the neonatal unit. Therefore, a location in close proximity or even inside the boundaries of neonatal unit is desirable. This also helps in administrative supervision by medical staff. Presence of human milk banks in the neonatal units is associated with elevated rates of exclusive breastfeeding rates in VLBW babies. Postnatal wards or Well Baby clinics of large hospitals are most suited for the purpose as donors are likely to be found in large statistics where health professionals can encourage them to donate milk.

\section{The recipients}

PDHM can be prescribed on priority for preterm babies and sick babies, babies of mothers with postpartum illnesses, and babies whose mothers have lactation failure, till their milk output improves. Therapeutic benefits of breast milk are noted in short gut syndrome, sepsis, and post-surgical gut healing in omphalocele, gastroschisis, bowel obstruction and intestinal fistulas. In extremely preterm infants given exclusive diets of preterm formula versus human milk, there was a significantly greater duration of parenteral nutrition and higher rate of surgical necrotizing enterocolitis (NEC) in infants receiving preterm formula.

If PDHM supplies are sufficient, donor milk may be supplied for:

- Absent or insufficient lactation

- For babies of non-lactating mothers

- Abandoned neonates and sick neonates 
- Temporary interruption of breastfeeding

- Infant at health risk from breast milk of the biological mother

- Babies whose mother died in the immediate postpartum period

\section{Infrastructure}

There are no standard recommendations or exact guidelines mentioned concerning the space necessities for construction of human milk banks. The minimum requirement is a partitioned room of 250 square feets that can contentedly lodge at least the equipment necessary for milk banking, a work area for the technician as well as some storeroom for records, management and area for counseling donors etc. Privacy is of supreme significance for area of breast milk expression.

\section{Equipments}

Pasteurizer/Shaker-water bath: It is vital to have a appliance to carry out heat action of donor milk at the recommended temperature of $62.5^{\circ} \mathrm{C}$ for a period of 30 minutes prior to its use. The milk in the container is boiled through the steam and hot water in the water shaker bath. To avoid coagulation of the milk and to dispense heat uniformly, the tray on which the milk containers are placed is shaken/wobbled. This shaker water bath should be double walled and made of steel. Its size varies according to the need of the milk bank, with the tray capacity varying from 9 to 24 containers of 200 to $400 \mathrm{~mL}$ capacity.

Deep freezer: A deep freezer to store the milk at $-20^{\circ} \mathrm{C}$ is essential in the milk bank. It is desirable to have two deep freezers with a digital display of the temperature inside it with an alarm setting. First for storage of the milk till the post-pasteurization milk culture reports are available. This freezer should be locked at all times with access only to the technician, so that no milk is accidentally used till the culture reports are on hand. The second deep freezer is used for storage of the pasteurized milk once the culture reports are negative and the milk is considered safe for disbursement.

Refrigerators: These are required to store the milk till the whole day's collection is over and the milk is ready to be mixed and pooled for further processing. It is also required for thawing the milk before being dispatched. Preferably two different units should be used for these purposes.

Hot air oven/Autoclave: A hot air oven/autoclave in the milk bank or centralized sterile service department is necessary for sterilizing the containers used for gathering from donors, containers for pasteurization and the test tubes wanted for sending milk culture samples to the microbiology laboratory.

Breast milk pumps: For milk banking, hospital grade electric pumps are favored as they result in better volumes of expressed milks and are relatively painless and at ease to use. Manually operated breast milk pumps intended to operate more physiologically by simulating the infant's compressive action on the areola during breastfeeding can be used with lower cost implications. They can be reused with chemical disinfection/sterilization.

Containers: For collection and storing the milk, single use hard plastic containers of polycarbonates, pyrex or propylene are used across the world. Polythene milk bags are not suitable as they are fragile, associated with loss of lipids and vitamins and there is a risk of contamination, although some studies have challenged the loss of lipids.
Generator: Every milk bank should have a dedicated centralized source of uninterrupted power supply backup to run the deep freezers and refrigerators in case of electricity failure.

Milk analyzer: It is desirable to have macronutrient analysis of breast milk to estimate the calorie, protein and fat of a milk sample, using infra-red spectroscopy technology, in teaching hospitals as a step towards lacto- engineering.

\section{Administrative staff}

Human milk banks should have a panel of consultants to guide overall development and functioning. It can include representatives from the areas of pediatrics/neonatology, lactation, microbiology, nutrition, public health and food technology. It should consist of a Director (for planning, implementing and evaluating the services), milk bank officer (usually a doctor), Lactation management nurses (for counseling mothers and assisting expression of breast milk), milk bank technician (for pasteurization of breast milk and microbiological surveillance), Milk bank attendant (for collecting, sterilization of the containers and maintaining hygiene), receptionist (for record keeping and public relations) and a microbiologist (for microbiology testing and infection control policies).

Guidelines for staff:

- Standard operating procedures (SOP) of the bank (which should be displayed at proper places) should be adhered to.

- Hygienic practices like proper hand wash, donning gowns, mask, gloves, trimming nails, locking long hairs should be maintained.

- Gloves should be worn and changed between handling raw and heat-treated milk.

- Staff should undertake regular health checks and be immunized against Hepatitis B.

- There should be a program for constant training of the staff.

\section{Donor population}

The donor population is formed by healthy lactating mothers with healthy babies, who are voluntarily willing to give their extra breast milk for other babies without compromising the nutritional needs of their own baby. The donors can include mothers attending well baby clinics, mothers whose babies are in neonatal intensive care units, those who have lost their babies but are willing to donate their milk, or lactating working staff in the hospital, and motivated mothers from the community. Donors are not paid for their donations.

\section{Who can donate?}

A lactating woman who:

- Is in good health, good health-related behavior, and not regularly on medications or herbal supplements (with the exception of prenatal vitamins, human insulin, thyroid replacement hormones, nasal sprays, asthma inhalers, topical treatments, eye drops, progestin-only or low dose estrogen birth control products);

- Is willing to undergo blood testing for screening of infections; and

- Has enough milk after feeding her baby satisfactorily and baby is thriving nicely. 


\section{Who cannot donate?}

A donor is disqualified who:

- Uses illegal drugs, tobacco products or nicotine replacement therapy; or

- Regularly takes more than two ounces of alcohol or its equivalent or three caffeinated drinks per day; or

- Has a positive blood test result for HIV, HTLV, Hepatitis B or C or syphilis; or

- Is herself or has a sexual partner suffering from HBV, HIV, $\mathrm{HCV}$ and venereal diseases OR either one has high risk behavior for contracting them in last 12 months; or

- Has received organ or tissue transplant, any blood transfusion within the prior 12 months.

- Is taking radioactive or other drugs or has chemical environmental exposure or over the counter prescriptions or mega doses of vitamins, which are known to be toxic to the neonate and excreted in breast milk or

- Has mastitis or fungal infection of the nipple or areola, active herpes simplex or varicella zoster infections in the mammary or thoracic region.

\section{Collection of breast milk}

After proper counseling, checking appropriateness for donation, getting written informed consent, history taking, physical examination and sampling for laboratory tests, the donor is sent to selected breast milk collection area in the milk bank or in the milk collection center Breast milk is collected by trained staff with hygienic precautions. Washing the breast with water before expression is as good as washing with disinfectant. There is no underlying principle in disposal of foremilk. The breast milk may be expressed manually or with breast pumps. Manual expression is a low cost and effective method of expression, and associated with less risk of contamination. Simultaneous breast expression in breastfeeding women is more efficacious than sequential breast expression. Milk should be collected in properly labeled sterile container and transported to HMB under cold storage condition.

\section{Processing}

All collected raw breast milk should be refrigerated immediately till the serological report comes negative. While mixing fresh raw breast milk to frozen raw breast milk previously collected from same donor, it should be chilled before adding to frozen milk. For sick or preterm babies, it is wise to use a new bottle for each pumping. Before pasteurization, pooling and mixing may be carried out from multiple donors to simplicity the process of processing and storage. Microbiological screening of donor milk is done before and as soon as possible after pasteurization. Even after pasteurization, the endotoxins of organisms are still present in the milk in some cases but they have not been found to have any clinical effect on the baby. A bacterial count of $105 \mathrm{CFU} / \mathrm{mL}$ or more in raw breast milk can be an indicator of the poor quality of milk. Whole batch of culture positive container of pasteurized milk should be discarded.

\section{Storage}

Pasteurized milk awaiting culture report should be kept in freezer area taking precaution not to disburse it till the culture is negative. Storage should be done in the same container that is used for pasteurization. Culture negative processed milk should be kept at $-20^{\circ} \mathrm{C}$ in tightly sealed container with clear mention of expiry date and other relevant data on the label. It can be preserved for 3 to 6 months.

\section{Disbursal}

PDHM should be disbursed at physician's requisition from NICU physician after informed consent from the parents of the recipient. Preterm baby should preferably get PDHM from preterm donors. It should be done on First-in-first-out basis from the storage. Transport of PDHM should be done under cold storage in the same pasteurized container till its use. Frozen PDHM should be thawed by either defrosting the milk rapidly in a water bath at a temperature not exceeding $37^{\circ} \mathrm{C}$, or under running lukewarm water taking care that the cap of the container does not come in contact with the water as it is likely to get contaminated. It should never be thawed in a microwave as this result in reduction in the $\operatorname{IgA}$ content of the milk and there is a risk of burns if the milk is used too soon. Milk should not be refrozen after being thawed as this increases the hydrolysis of the triglycerides in the milk. While bringing to room temperature, it should be gently agitated to make a homogenous mixture before use and should be used preferably within 3 hours to prevent contamination.

\section{Labeling and record keeping}

Secrecy of records should be maintained by the milk bank. Proper labeling at all levels is compulsory; from sterile container for collection of donation, pooling vessel and pasteurization container to storage containers. Labels should be water resistant and names and identification information of donors, dates of pasteurization, batch numbers and expiry date should be clearly legible. Record keeping at all levels should be meticulous for Donor Record File containing consent form, donor's and her child's data, screening reports, pasteurization batch files, and for PDHM Disbursal Record File containing relevant data, including recipient consent form.

\section{Conclusion}

It is clear that artificial formula will never offer the broad variety of benefits of human milk. Given the high rate of preterm births in the country and level of malnutrition that ensues in the postnatal growth in such babies after birth, there is a burning need to set up milk banks crosswise the country, particularly in the large neonatal units of all hospitals.

\section{Acknowledgments}

None.

\section{Conflicts of interest}

Author declares that there is none of the conflicts. 